\title{
Tonsils Increase in Weight during Growth
}

\author{
Susumu Mukai \\ Otorhinolaryngology, Mukai Clinic, Yamato, Kanagawa, Japan. \\ Email: s-mukai@da2.so-net.ne.jp \\ Received January $18^{\text {th }}, 2013$; revised March $10^{\text {th }}, 2013$; accepted April $14^{\text {th }}, 2013$ \\ Copyright (C) 2013 Susumu Mukai. This is an open access article distributed under the Creative Commons Attribution License, which \\ permits unrestricted use, distribution, and reproduction in any medium, provided the original work is properly cited.
}

\begin{abstract}
Purpose: During my 40 years of performing tonsillectomy, I have noticed that tonsils seem to increase in size with aging. The purpose was to study the relationship between the weight of tonsils and patients' age. Subjects and Methods: Tonsillectomies were performed in 1246 patients diagnosed with sleep disorders with hypertrophied tonsils. The excised tonsils were weighed. Results: 1) The number of male tonsillectomy cases was 2.3 times higher than the number of female cases; 2) Most tonsillectomies were performed in children three to six years of age, and in adults who were around 30 years old; 3) The maximum age of the male patients was 57 years and that of the female patients was 63 years; 4) The weight of tonsils increased with age in the males, but the maximum weight of female tonsils was reached at around nine years of age; 5) Tonsils of males were heavier and larger than those of females of the same age; 6) A high degree of correlation was observed between the weights of the right and left tonsils of a subject. Conclusions: 1) The weight of tonsils increased with growth; 2) Tonsils in males were more hypertrophic than those in females; 3) The age distribution of the cases suggested that the lifespan of these patients is shorter than the norm; 4) The ideal age at which to perform tonsillectomy is thought in two to three years; 5) A high degree of corelation eixists between the weight of the right and left tonsils of a subject.
\end{abstract}

Keywords: Sleep Disorder; Gender; Lifespan; Tonsillectomy; Development

\section{Introduction and Purpose}

Tonsillar hypertrophy is diagnosed clinically when tonsils protrude from the oral anterior pillars. However, it is not rare that at the first inspection both tonsils protrude from the anterior pillars and almost touch each other. After tonsillectomy, the tonsils are not as large as suspected at the first inspection. The actual size of tonsils cannot be determined only from inspection of tonsils in the mouth [1-4]. The real volume and weight of a tonsil can be appreciated only after tonsillectomy. A heavy tonsil is large. Clinical tonsillar hypertrophy comprises both relatively bulky tonsils and heavy tonsils [5, $6]$.

During inspection of tonsils, I observe the tonsils, oropharynx, hypopharynx, epiglottis and larynx by laryngofiberscope via the nose. Fiberscopes make it possible to examine large buried tonsils and tonsils that are enlarged at their lower poles. In addition, buried hypertrophied tonsils are palpable from the outside on the neck under the angle of the lower jaw.

Patients with hypertrophied tonsils do not feel that their breathing is difficult. Sometimes hypertrophied ton- sils are attached to each other in the fauces. The choanae in both infants and adults are narrow, and fiberscopic observation via the nose shows that the epiglottis and larynx are hidden by tonsil protrusions. I often wonder how patients can breathe under those obstructed conditions. These patients, especially infants, subconsciously alleviate their breathing difficulties caused by their hypertrophied tonsils, by sleeping on their hands and knees similar to the way in which a puppy sleeps by burying its head between the paws. They find comfortable positions during sleep. Tonsillar hypertrophy is like a congenital disease. Patients do not feel that the tonsillar hypertrophy obstructs their breathing. The fact that patients roll about while asleep means that they struggle against dyspnea. These sleeping positions and the fact that they turn while asleep imply that their lives are threatened.

Sometimes patients' families think that rolling while asleep and/or moving around is characteristic of infants. Adult patients with tonsillar hypertrophy also show complete indifference to their disturbed sleep. Although they have many symptoms of tonsillar hypertrophy mentioned above, they think that they can sleep well. 


\section{Cases and Methods}

\subsection{Cases and Duration of Observation}

This study included patients who were diagnosed with tonsillar hypertrophy and underwent tonsillectomy at Otorhinolaryngology, Mukai Clinic during the 11-year period between June 27, 2000 and June 14, 2011. This prospective study was approved by the Human Subjects Committee of our clinic. Informed consent was obtained from each patient or guardian.

\subsection{Tonsillectomy}

Tonsillectomies were performed under general anesthesia in patients who were under 12 years old, and under local anesthesia in those 12 years old and over. Tonsillectomy under general anesthesia was performed using Mukai's tonsillectomy method [7]. According to this procedure, local anesthetics are applied to tonsillar capsules under general anesthesia, i.e., lidocaine to which is added epinephrine is injected under the tonsillar capsules, and the tonsils float to the tonsillar beds as a result. Ablation of tonsils becomes easier when they are floating, and bleeding is minimized by contraction of the vessels by the epinephrine contained in the local anesthetic. As a result, the operation time is shorter and the depth of the general anesthesia is shallow. Patients wake up shortly after completion of the operation.

From June 27, 2000 to May 2004, a semiconductor LASER knife (MEDILASER 20L, MATSUSHITA ELECTRIC Co. Ltd., Tokyo, Japan) and from June 2004 a high-frequency radio knife (Elleman Japan Co. Ltd., Tokyo, Japan) were employed for tonsillectomies. The duration of the tonsillectomy itself was within $15 \mathrm{~min}-$ utes as performed with either of the two knives.

\subsection{Measurement}

The excised tonsils were weighed on a digital scale (YUYAMA CS-300S, Yuyama Ltd., Osaka, Japan; minimum weight $0.01 \mathrm{~g}$ ) as soon as they were detached.

StatView and Exel were employed for statistical analysis.

\section{Results}

\subsection{Cases}

1246 patients (male 871, female 375 ) underwent tonsillectomy during the 11-year study period. There were 2.3 times more males than females (Figure 1).

\subsection{Age Distribution}

The patients' ages ranged from 1 to 63 years. The cases were classified according to age. Patients with ages ranging from 1 to less than 10 years were grouped in one-year groups: the group comprising patients from one to less than two years old was designated as $1 \leq$ case $<2$, the group of those from 2 to less than 3 years old as $2 \leq$ case $<3$ and so on until $9 \leq$ case $<10$.

Beyond 10 years of age, the patients were grouped as $10 \leq$ case $<15,15 \leq$ case $<20,20 \leq$ case $<30,30 \leq$ case $<40,40 \leq$ case $<50,50 \leq$ case $<60$, and $60 \leq$ case (Figure 2).

Among those under 15 years of age, the group with the largest number of cases was the $5 \leq \mathrm{y}<6$ group with 146 cases, followed by the $4 \leq \mathrm{y}<5,3 \leq \mathrm{y}<4,10 \leq \mathrm{y}<15$ and $6 \leq \mathrm{y}<7$ groups with 125, 117, 105 and 99 cases, respectively. There were a total of 841 cases under 15 years of age.

Among patients over 15 years of age, most of the cases were in the $30 \leq$ case $<40$ group (184 cases), followed by the $20 \leq$ case $<30,40 \leq$ case $<50,15 \leq$ case $<20$ and $50 \leq$ case $<60$ age groups with $86,81,28$ and 23 cases, respectively. Only three female cases were over 60 years old (Figure 2).

\subsection{Tonsil Weight}

A total of 2492 tonsils from 1246 patients were weighed.

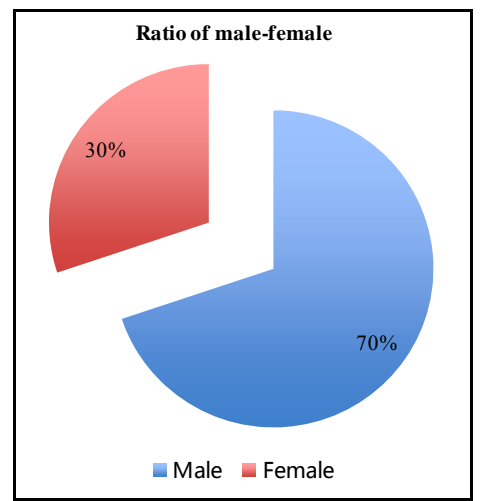

Figure 1. Male-female ratio in 1246 tonsillectomy cases (male 871/70\%, female $375 / 30 \%$ ).

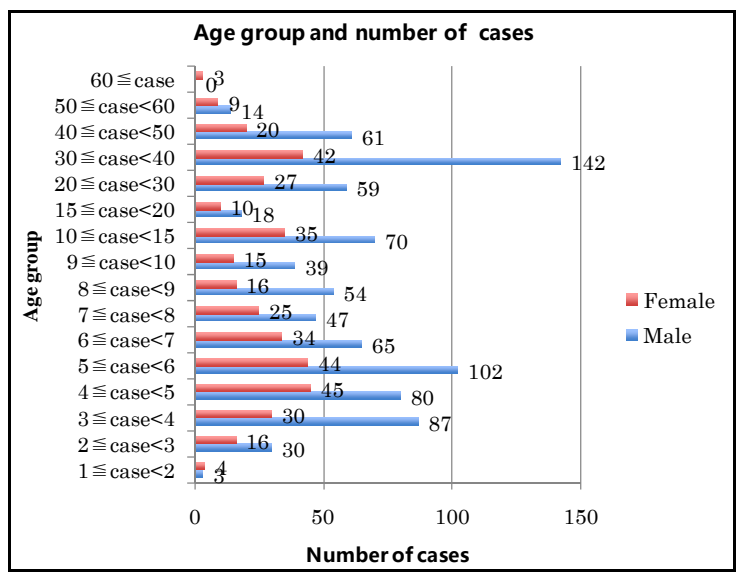

Figure 2. Age groups and number of cases. 
The mean weight of tonsils in males increased from $2.1 \mathrm{~g}$ at 1 year of age to $5.9 \mathrm{~g}$ at approximately 20 years of age, corresponding to body growth. The maximum mean tonsil weight in females was reached at 9 years of age. After that, no significant growth was observed in the tonsils of females (Figure 3).

A significant difference was observed between the weights of the male and female tonsils $(p=0.0005)$. The tonsils of males were heavier than those of females of the same age.

The minimum tonsil weight was $1.1 \mathrm{~g}$ (male, 3 years old, $14 \mathrm{~kg}$ ). The maximum tonsil weight in males was $13.5 \mathrm{~g}$ (right) and 12.5 (left), for a total of $26.0 \mathrm{~g}$ in a male weighing $100 \mathrm{~kg}$. The maximum tonsil weight in females was $8.0 \mathrm{~g}$ (right) and $13.7 \mathrm{~g}$ (left) for a total of $21.7 \mathrm{~g}$ in a female weighing $76.4 \mathrm{~kg}$.

\subsection{Relationship between Body Weight and Tonsil Weight}

The relationship between body weight and tonsil weight was investigated in infants between the ages of one and ten years $(\mathrm{n}=736)$ and in adults aged over 20 years $(\mathrm{n}=$ 377).

The body weights of infants were grouped into seven categories: under $13 \mathrm{~kg}(\mathrm{w}<13)$, from $13 \mathrm{~kg}$ to $16 \mathrm{~kg}$ $(13 \leq \mathrm{w}<16), 16 \leq \mathrm{w}<19,19 \leq \mathrm{w}<22,22 \leq \mathrm{w}<25,25$ $\leq \mathrm{w}<28$, and $28 \mathrm{~kg} \leq \mathrm{w}$ (Figure 4). In boys, an increased body weight resulted in increased tonsil weight. In contrast, very low increases in girls' tonsil weights were observed corresponding to body weight increases from $13 \leq \mathrm{w}<16 \mathrm{~kg}, 16 \leq \mathrm{w}<19 \mathrm{~kg}$, to $19 \leq \mathrm{w}<22 \mathrm{~kg}$. A significant difference was observed between boys' and girls' tonsil weight/body weight ratios $(\mathrm{p}<0.01)$.

The body weights of adults were divided into 6 groups: under $50 \mathrm{~kg}(\mathrm{w} \leq 50), 50-60 \mathrm{~kg}(50 \leq \mathrm{w}<60), 60-70$ $\mathrm{kg}(60 \leq \mathrm{w}<70), 70-80 \mathrm{~kg}(70 \leq \mathrm{w}<80), 80-90 \mathrm{~kg}$ $(80 \leq \mathrm{y}<90)$, and over $90 \mathrm{~kg}(90 \leq \mathrm{W})$ (Figure 5).

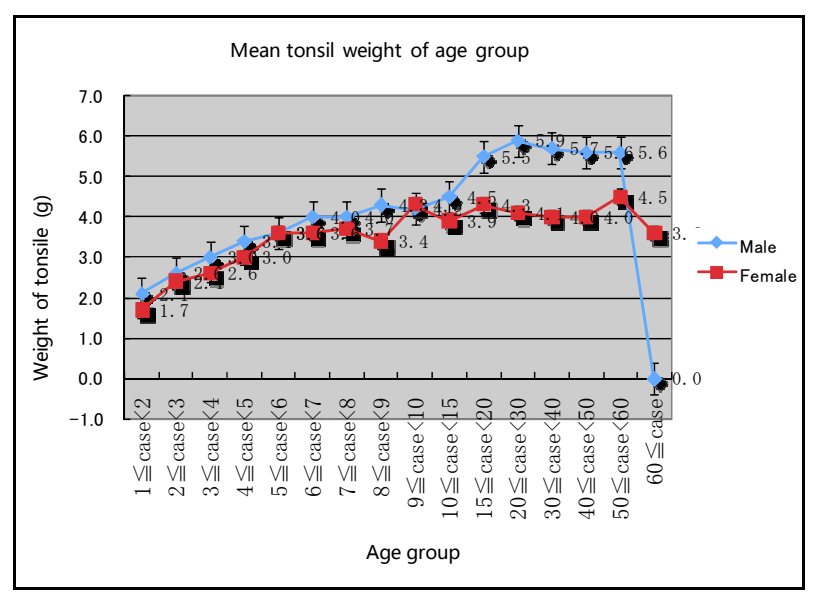

Figure 3. Mean tonsil weights of age groups.

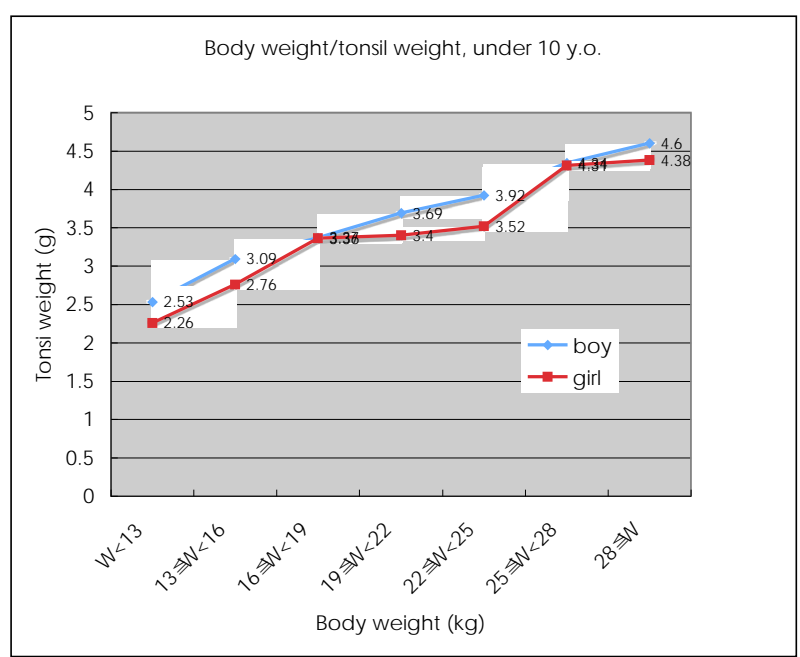

Figure 4. Relationship between body weight and tonsil weight under 10 years old.

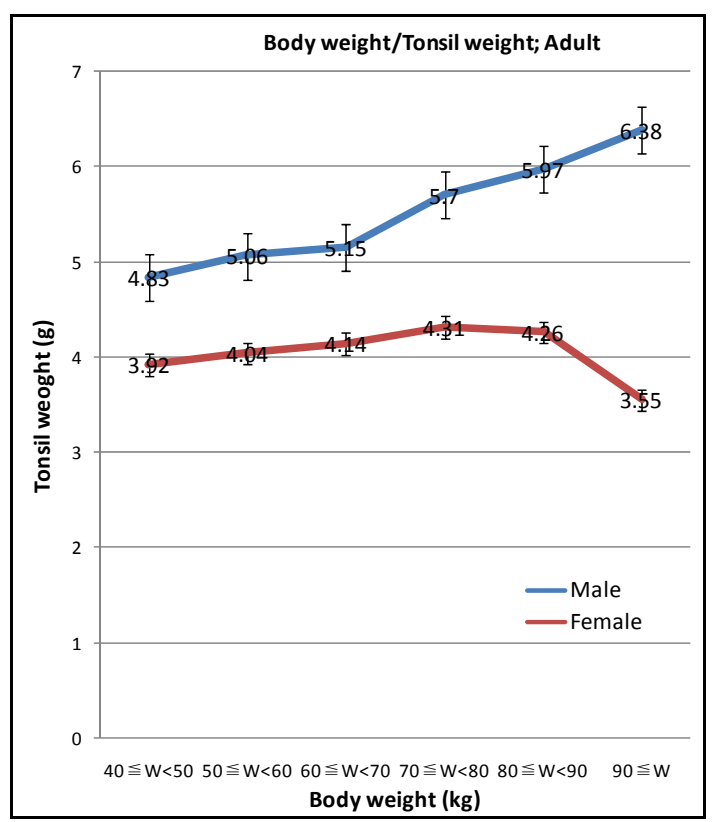

Figure 5. Relationship between body weight and tonsil weight of adult $(p=0.0042)$.

The tonsils of males were heavier than those of females in every weight group. A statistically significant difference was observed between male and female tonsil weight/body weight ratios $(p=0.0042)$.

In males, tonsil weight increased in accordance with increases in body weight. The coefficient of correlation between male body weight and tonsil weight was $\mathrm{R}=$ 0.2421 .

No such correlation trend was observed between tonsil weight and body weight in females. The coefficient of correlation between female body weight and tonsil weight was $\mathrm{R}=0.0900$. Thus, there was a poor relationship between female body weight and tonsil weight. 


\subsection{Correlation between Right and Left Tonsils in Each Subjects}

The distribution of the right and left tonsil weights is shown in Figure 6. There was a high degree of correlation between the weights of the right and left tonsils in a subject $(\mathrm{R}=0.896941)$, showing that the right and left tonsils grow similarly in a subject (Figure 6). The average weight of the right and left tonsils was employed as an individual's tonsil weight in this paper.

\section{Discussion}

This investigation revealed that tonsil weight increased with growth of the individual and that there was a difference in tonsil weight between the genders. The observed evidences were as follows.

\subsection{Male-to-Female Ratio}

Among the 1246 cases who underwent tonsillectomy, 871 $(70 \%)$ were male, indicating that there were 2.3 times more male than female cases. This suggests that male tonsils are more apt to hypertrophy than those of females.

\subsection{Sex Difference in Tonsillar Hypertrophy}

Tonsillectomies were performed 2.3 times more frequently in males than in females. In addition, the tonsil weight among males increased from $2.1 \mathrm{~g}$ in a one-yearold male to $5.9 \mathrm{~g}$ in a male of approximately 20 years. In contrast, the maximum tonsil weight in females was reached at around 9 years of age. After 9 years of age, a significant increase in tonsil weight was not observed.

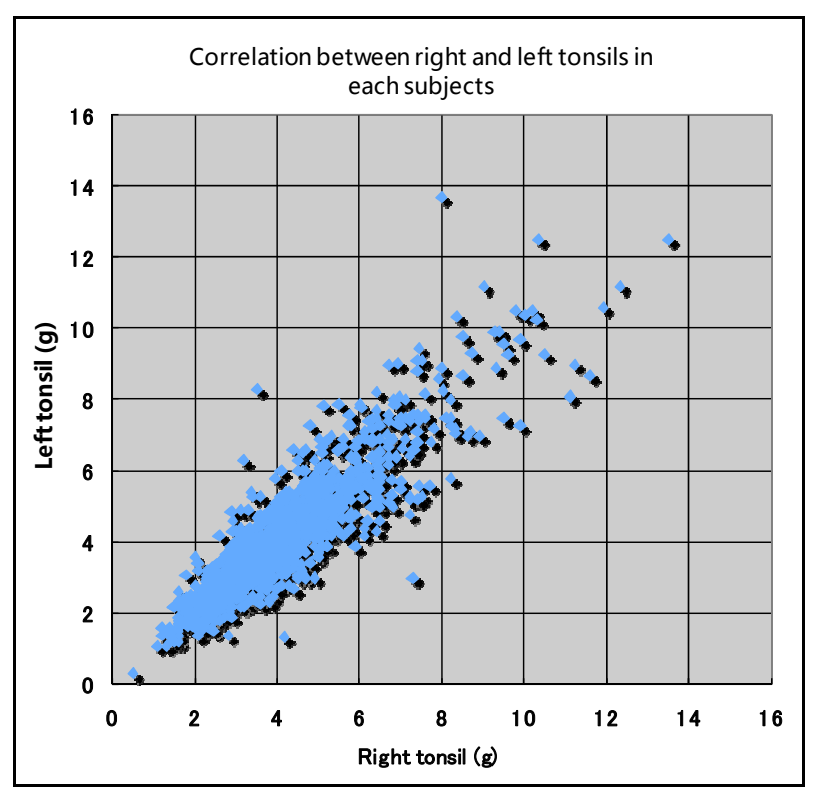

Figure 6. Correlation between right and left tonsils in each subjects $(n=1246)$ (There were significant relationships between right and left tonsils in a subject. $R=0.896941$ ).
This observation means that male tonsils continue to grow into adulthood, but female tonsils remained prepubescent.

A statistically significant difference was observed in tonsil weight between males and females $(p<0.0005)$. In individual age groups, tonsils in males were heavier than those in females.

Male tonsil weight increased proportionally with body weight. Statistically, there were significant differences in body weight/tonsil weight ratios between males and females in both the infant and adult groups. Moreover, the correlation coefficient between body weight and tonsil weight in males was $\mathrm{R}=0.2421$, and that in females was $\mathrm{R}=0.0090$. Thus, there was hardly any relationship between body weight and tonsil weight among females.

These results revealed that female tonsils are not prone to hypertrophy. In contrast, males showed a high frequency of tonsillar hypertrophy, and the tonsil weight was heavier and tonsils were more susceptible to hypertrophy. These results demonstrated that females have less tendency to develop OSAS than do males. This may be one reason that the average life span of females is longer than that of males.

\subsection{Age Distribution}

The age of the subjects who underwent tonsillectomy ranged from 1 to 63 years. The age group with the largest number of cases under 15 years old was the $5 \leqq y<6$ group with 146 cases. The adenoids increase in size during this period. Hypertrophy of tonsils and adenoids interferes with respiration and sleep. At the time when children start school, atrophy of adenoids as well as growth of the maxilla and mandible bones makes respiration easier. These conditions seem to reduce the necessity of tonsillectomy.

In adults, the largest number of tonsillectomies was carried out in the $30 \leq$ case $<40$ group. Symptoms of OSAS develop most frequently in this age group; they interfere with the patients' lives and cause them to seek medical advice.

The rate of tonsillectomy decreased significantly at around 50 years of age. Among those over 60 years of age, there were no male cases and only three female cases. This does not mean that the hypertrophied tonsils shrink at this age. Instead, it means that the life span of patients with tonsillar hypertrophy is limited to approximately 60 years.

\subsection{Correlation between Right and Left Tonsil Weight}

Two reports have been published on right and left tonsil weights. Both of them reported that there was no difference between right and left tonsil weights [8,9]. Al- 
though there were no pathological malignancies in the tonsils in the present study, in the study of Beaty et al. [10], out of 25 malignant tonsils, 20 had tonsillar asymmetry.

In the present study, there was a high degree of correlation between the right and left tonsil weights in individual subjects $(\mathrm{R}=0.896941)$. This high degree of correlation means that both tonsils grow in correlation with each other.

\subsection{The Advisable Age for Tonsillectomy}

During my 40-year experience of treating patients with tonsillectomy, I have observed that many children with tonsillar hypertrophy have the following symptoms: Tonsillar hypertrophy is accompanied by adenoid hypertrophy. The mouths of children are usually open due to adeno tonsillar hypertrophies and they have halitosis. They frequently get sinusitis and otitis media.

They have no or very narrow interdental spaces and hypoplastic maxillo-mandibular bones, and they could not fully open their mouths. They had thin chests and sometimes increased resistance in the upper respiratory tract, resulting in concave front chest walls at the attachment of the diaphragm.

They roll about while asleep, have enuresis, move aimlessly around while taking meals, cannot remain sitting on the chair while taking classes, have poor memory, are underdeveloped and lack a will to learn.

It is not rare that hypertrophy of the heart and/or aerophagia is observable by chest X-ray. These symptoms are associated with respiratory insufficiency/sleep disorders in infants.

They are also emotionally disturbed, are very afraid of being examined and are unruly. They are awkward and unstable.

Symptoms of tonsillar hypertrophy in adults are as follows: fatigue, sleepiness in the daytime, frequent tonsillorespiratory infection and sometimes peri-tonsillar abcess, sleep disorders, snoring and obstructive sleep apnea [11]. In addition, they have a tendency to be obese, and have a swelled abdomen and fatty liver.

Tonsillectomy needs to be performed before these signs and symptoms distinctly develop. Historically patients resist infectious diseases after tonsillectomies [4, 12]. Tonsillectomy brings little effect on the human immune functions. The age at which tonsillectomy should be performed is 2 or 3 years. For a five-year-old child, the span from two years old to five years old is physiologically double and a half. In addition, infancy is a very important social learning period. If tonsillectomy was performed at two years old for a five-year-old child, the next double and a half of his life would be free from tonsillar hypertrophy.

Furthermore, it is easier to perform tonsillectomy on children of 2 to 3 years of age because there is little adhesion of the tonsils with tonsillar beds. Ablation of tonsils from their beds can be easily performed, causes little bleeding and is safe at this young age.

Children who had received tonsillectomy at the age of 2 or 3 years have good communication with others. They frequently laugh and move around full of energy. Tonsillectomy at these ages brought good results in their lives.

When infants are diagnosed with tonsillar hypertrophy, tonsillectomy should be performed with no hesitation.

Living with tonsillar hypertrophy until adulthood may result in learning problems, emotional disorders and lack of sociability. The rates of ischemic heart disease, apoplexy, arrhythmia and traffic accident are high, and the mortality rate in the age group less than 50 years old is high among OSAS cases [13].

\section{Conclusions}

The weight of tonsils increased with growth. Tonsils in males were more hypertrophic than those in females. The age distribution of the cases suggested that the lifespan of these patients is shorter than the norm.

A high degree of corelation eixists between the weight of the right and left tonsils of a subject.

In patients with tonsillar hypertrophy, the ideal age at which to perform tonsillectomy is two to three years. If tonsillectomy is not performed at that age, it should be done when the patient is diagnosed with tonsillar hypertrophy.

\section{REFERENCES}

[1] M. Makenzie, "Tonsile Disease of the Throat, Nose and Ear,” C. V. Mosby Co., St Louis, 1920, pp. 24-51.

[2] M. Makenzie, "Chronic Hypertrophy of the Tonsils," In: M. Makenzie, Ed., Diseases of the Throat, Nose and Ear, William Heinemann, London, 1927, pp. 50-62.

[3] M. Sasaki, "Tonsillectomy and Its Indications," J ORL Soc Jpn, Vol. 47, 1941, pp. 1626-1648 (in Japanese).

[4] H. Toyota, "View from Tonsil Hypertrophy (On the Base of Tonsil Weight)," Jyuzen, Vol. 48, 1943, pp. 2236-2240 (in Japanese).

[5] E. Miwa, "Changes of Tonsils by Aging," Nagoya Medical Journal, Vol. 11, 1961, pp. 962-986 (in Japanese).

[6] N. F. Weir, "Clinical Interpretation of Tonsillar Size," Journal of Laryngology and Otology, Vol. 86, No. 11, 1972, pp. 1137-1144. doi:10.1017/S0022215100076325

[7] S. Mukai, M. Nagano, H. Fukuda, N. Shishikura and T. Tabata, "Use of Epinephrine-Added Local Anesthetic with Halothane Anesthesia in Tonsillectomy," J Otorhinolaryngol Japan (Igakushoin), Vol. 51, 1979, pp. 365369.

[8] H. Kitayane, "Clinical and Experimental Study on Tonsil Weight and Diameter Related to the Age, 2," Chuo-Igaku, Vol. 10, 1941, pp. 693-711 (in Japanese). 
[9] S. Yamamoto, "Tonsil Weight and Relationship with It's Embedding Part," Pract ORL Kyoto, Vol. 49, 1956, pp. 774-777.

[10] M. M. Beaty, G. F. Funk, et al., "Risk Factors for Malignancy in Adult Tonsils," Head \& Neck, Vol. 20, 1998, pp. 399-403.

[11] S. Mukai, N. Nitta, J. Hirano, et al., "Relationship between ADEL and Tonsillar Hypertrophy-Main Cause of Tonsillar Hypertrophy Is Depression of Respiration in In- fancy," Zetuyuchakusho Kenkyuukai Kaiho, Vol. 10, 2000, pp. 45-50 (in Japanese).

[12] Y. Honda, "Editorial: Consider about Tonsil Problems of the Elementary School Children," Pract ORL Kyoto, Vol. 32, 1937, pp. 739-744 (in Japanese).

[13] S. Sakurai, "Prognosis of Obstructive Sleep Apnea Syndrome-Evidences of Cardiovascular and Life Span," Journal of Clinical and Experimental Medicine, Vol. 216, 2005, pp. 629-632. 